\title{
Design and Fabrication and Hydrodynamic Analysis of a Fish Robot for Underwater Surveillance
}

\author{
Saeid Hosseini ${ }^{1}$, Kasra Daryani Tabrizi ${ }^{1 *}$, Ali Meghdari ${ }^{2}$ \\ ${ }^{I}$ School of Science and Engineering, Sharif University of Technology International Campus; Kish, Iran \\ ${ }^{2}$ School of Science and Engineering, Sharif University of Technology Tehran, Iran
}

*Corresponding Author: Kasra Daryani Tabrizi, School of Science and Engineering, Sharif University of Technology International Campus; Kish, Iran

\begin{abstract}
Condition and performance monitoring of underwater equipment and infrastructure is of one the major concern for marine industries. In the past two decades, several technique and methods have been developed for efficient monitoring of marine structures. Fish robots potentially can be used as an efficient device for under water monitoring.

The design is aimed at high maneuverability, efficiency and the ability to control velocity suitable for underwater surveillance. To control this robotic fish, a four-channel radio controller with $40 \mathrm{kHz}$ frequency, was used. Swimming mode of this robotic fish is categorized in carangiform swimming mode that use its tail and peduncle for propulsion and is faster, and has better maneuverability compared to its counterparts. It should be noted that the flexible tail could enhance the efficiency of the robotic fish swimming.
\end{abstract}

Employing a servo motor, flexible tail and pulley and rope mechanism, the robotic fish was designed and tested. The experimental tests indicate the maneuverability and the smooth swimming of the robotic fish.To optimize the design and manufacturing of the robotic fish, the hydrodynamic forces and proper function of the body form under water were analyzed using ANSYS fluent and computational fluid dynamics (CFD) method.

The present Robotic Fish significantly enhance the performance and functionality of Robotic Fish for underwater surveillance.

Keywords: Fish robot, Flexible tail, Design and fabrication, Hydrodynamic analysis,Computationalfluid dynamics (CFD), User-defined function (UDF)

\section{INTRODUCTION}

Today, mankind has realized that his existence depends on the preservation of the environment and animals that the most important of which are water resources such as rivers and oceans. Therefore, we constantly need to ensure their protection. One of the best possible ways to observe the underwater world is through robotic fish. So far, many researchers have been conducted on how tooptimize the function of the robotic fish. In this project, only the mechanism of the fish body movements is considered. In fish swimming, there are mainly two types of propulsion, i.e., the Body and/or Caudal Fin (BCF) propulsion [1,2] and the Medium and/or Paired Fin (MPF) propulsion [3,4].Most of the fast swimmers adopt BCF, such as sailfish, tuna, pike, etc. In fact, around $85 \%$ of fish species swim in BCF form. Examples for MPF swimmers are manta ray and box fish. In robot fish development, high speed and high efficiency are the main pursuits. As a result, most of existing robot fish adopts BCF as the way to generate thrust.

In general, the flapping motion of the robotic fish which is made of BCF mechanism can be divided into 3 categories 1. Multi-linked structure [5,6] 2. Full-bodies flexible robotic fish [7,8] 3 . Manufactured robotic fish from SMA and PZT materials $[9,10]$. In the first category, the control mechanism of the fish tail movement is so complex and costly but its maneuverability is high. In the second category, wind and water powered actuators were used to manufacture the robotic fish and the structure of the fish body is complex and costly and there is no control over the swimming of the fish. In third category, the thrust force is low but the mechanism of the structure and control is so simple and low cost. In this project, the aim is manufacturing a low cost robotic fish with a simple control mechanism that is able to maneuver properly and possesses an acceptable thrust force. 
According to open literature, most of existing works on fish robot are focused on modeling and simulation. In the present work a fish robot has been designed and fabricated. Several experimental tests and studies have been developed and performed to show the performance and functionality of the proposed system.

\section{DESIGNE}

The robotic fish consists of two major functional components. These are:

The fish's soft tail for forward propulsion,

Actuator and control board for the tail in the waterproof unit (fish's body).

\subsection{Fish Tail}

Based on the results previously obtained using test models, we believe that the flexibility of the caudal fin is an important factor for improving the propulsive performance of robotic fish [10]. For this reason, the robot is equipped with a caudal fin made of a flexible material.

In order to optimize the geometry of the flexible tail, the width was copied from the geometry of a real fish, which was proposed by Alvarado \& Toumi, 2006 that shows in figure 1.

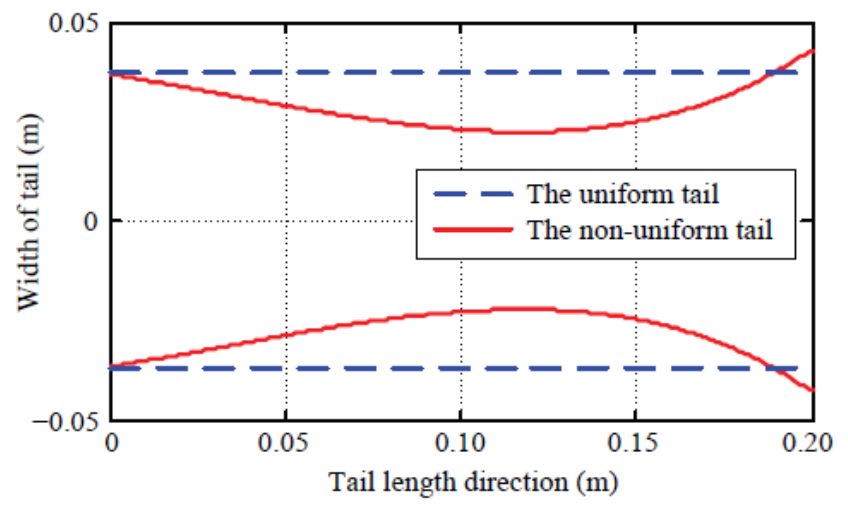

Figure1. Geometries of tails [11]

As shown in figure 2, the design of the first section of the robot fish tail was based on the following geometric shape and the second section of the robot fish tail was added to the first section in order to apply force and create moment. To do so, a servomotor and a cable connected to point $\mathrm{O}$ were used to create moment in tail and the pressure of water in the opposite direction of moment to the first section of the fish tail can create a wave like movement in the tail. Using this simple mechanism, we were able to create vortex in water and copy the mechanism of a real fish movements. Since the ratio of the first section curve is smaller than the second section, the dynamic analysis bending of the first tail in water is possible through Euler-Bernoulli beam theory [12] as

$\rho A(x) \frac{\partial^{2} h(x, t)}{\partial t^{2}}+\frac{\partial^{2}}{\partial x^{2}}\left[E I(x) \frac{\partial^{2} h(x, t)}{\partial x^{2}}\right]=F(x, t)+L_{y}(x, t)$

where $0<x<L$ and $L$ is the length of the tail; $\rho$ is the density of the material of the tail; $A(x)$ is the area of cross-section of the tail; $E$ is Young's modulus; and I( $x)$ is the moment of inertia of the area of the cross-section at $x$ about the bending axis. $\mathrm{EI}(\mathrm{x})$ is the bending stiffness of the beam.

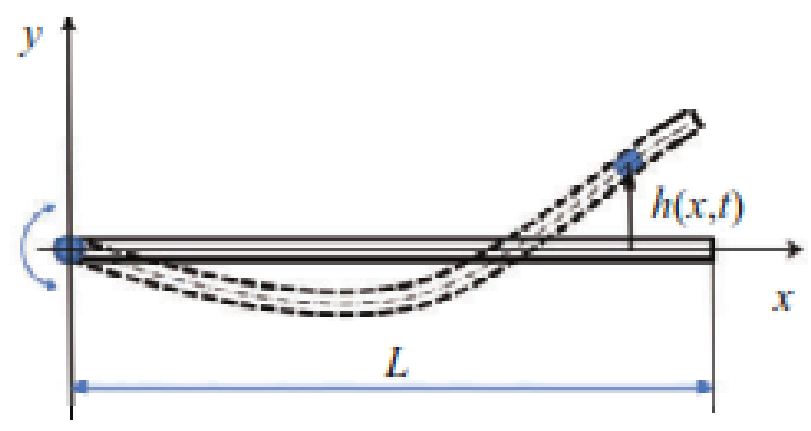

Figure2. Euler-Bernoulli beam theory 


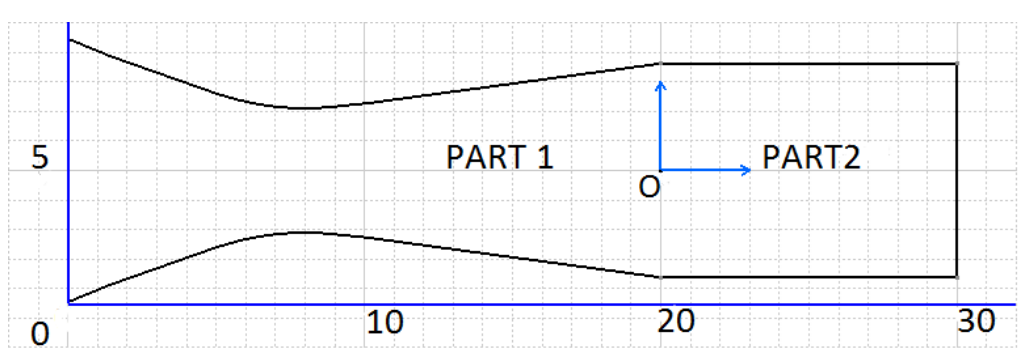

Figure3. Caudal fin design

\subsection{Actuator Selection}

The pressure of water in the opposite direction of moment to the first section of the fish tail can create a wave-like movement in the tail. Using this simple mechanism, we are able to create vortex in water and copy the mechanism of a real fish movements. Since the ratio of the first section curve is smaller than the second section, the dynamic analysis of the tail is possible through Bernoulli's law.

In past iterations of BCF propulsion mechanism, flexible tail was used with complicated motion design, but in this approach with a simple mechanism using cables and choosing the fish tail from an elastic material, the lateral movement for the tail with suitable force and exceptional speed was created.

\subsection{The Control System Architecture}

Microcontroller unit(MCU) sends Pulse-Width-Modulation (PWM) signal to the motor driver to control the tail motor's speed, and thus the ossillatory frequency of the tail is control by duty ratio of the PWM signal.

\section{FABRICATION}

\subsection{Material Selection}

To choose the right material for the fish tail, several factors are taken into consideration. Firstly, the material needs to be flexible to be able to spring back to the first position after bending so that it can create a wave-like movement of the fish tail while swimming. Secondly, the tail needs to be stiff enough to push the water and not too stiff so that water can have no effect on it. So for this reason High Density Polyethylene (HDPE) sheet with the thickness of $1 \mathrm{~mm}$ was used for the tail.

\subsection{Electrical Design}

For the actuation of the tail, a servomotor was selected. Since servos are fully self-contained, the velocity and angle control loops are very easy to implement, while prices remain very affordable. Servomotors are a conventional and proven technology, and many control platforms currently exist for their implementation.

To create a control signal for servomotor movement, a ATMEGA32 controller with timer of one second was used. The timer count Additive from 0 to the specific number and then downturn to 0 again. One may note that the system clock and clock timer speed are two important parameters.

\subsection{Body Construction and Sealing}

The head of the robot fish has been designed using solidwork and made by plastic injection technology.

In order to manufacture the fish tail, a sheet of HDPE (High Density Polyethylene) with the thickness of $1 \mathrm{~mm}$ was provided. Then, the pattern of a fish tail was printed and cut out of HDPE. After cutting the tail of the fish a metal fasteners were installed $10 \mathrm{~cm}$ far from the beginning of the tail.

All the electrical equipment (including the control board, radio transmitter, servo motor and battery) were installed on a $22 \times 9 \times 0.2$ aluminum plate. A disk with the radius of $11 \mathrm{~cm}$ was placed between the head and the tail of the robotic fish so that it can function as a support for the tail and the aluminum plate and also function as a sealing lid for the body of the fish. Two holes with the distance of $3 \mathrm{~cm}$ from the center of the disk and 0.01 diameters were drilled so that the cables can pass through them. The respective cables are connected to the pulley installed on the servo and the tail.The tail and the aluminum plate were clamped to each side of the disk using bolts and knots. 


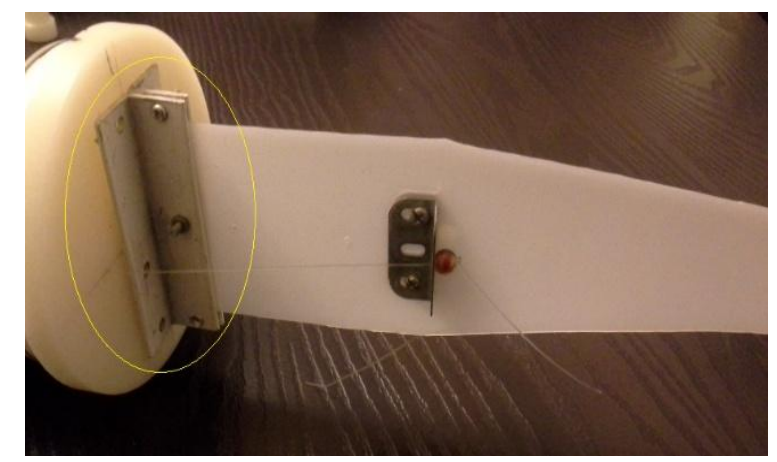

Figure4. connecting the tail to the disk

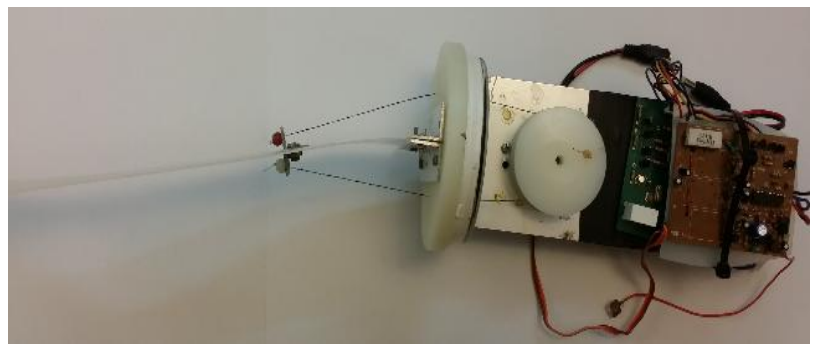

Figure5. The Mechanism of robotic fish actuation andmechanical parts assembly

A plastic cover is used to make the equipment waterproof. This plastic cover (with aerodynamic shape) was chosen and for balancing, Pectoral fins were designed based on a real fish and fabricated with $0.2 \mathrm{~cm}$ aluminum plate and fixed on the head of the robotic fish

\section{EXPERIMENTAL RESUlTS}

Given the fact that this robotic fish is designed for underwater surveillance, the radius within which the fish swims is of great importance and the less the radius the more efficient the robotic fish functions. The extent to which the fish tail bends, determines the radius of movement and the mechanism of the movement of the robotic fish is designed in a way which is capable of making a Uturn within the radius of 35 centimeters (Table 2). Compare to flexible tailed robotic fish ([7],[8],[9]), the mechanism to control the bending of the robotic fish tail is more efficient in terms of maneuverability.

\subsection{Effect of Changed Frequency of Flapping Motion}

The robotic fish was tested for frequencies from $0.5 \mathrm{HZ}$ to $2 \mathrm{HZ}$ and fixed amplitude of $7.5 \mathrm{~cm}$. the results is shown in table 1 . As it can be seen in table 1 , the speed increase until $f=1.25 \mathrm{HZ}$ but after that, the servomotor cannot handle the amplitude and it cause reduction in both speed and amplitude.



Figure6. Fabricated Robotic Fish

Table1. Forward swimming speed and tail's displacement at various frequencies and $A=7.5 \mathrm{~cm}$

\begin{tabular}{|c|c|}
\hline tail Frequency of actuation $(\mathrm{HZ})$ & Forward Swimming speed (cm/s) \\
\hline 0.5 & 12 \\
\hline 0.75 & 14.8 \\
\hline 1 & 18.1 \\
\hline 1.25 & 18.8 \\
\hline 1.5 & 18 \\
\hline
\end{tabular}




\subsection{Change Offset}

Changing the offset of the tail actuation, resulted in the robotic fish turning. The turning direction of the robot is opposite to the changing offset direction. Results of different offsets are presented in table 3 .

Table2. Turning performance of the robotic fish with different offsets af $F=0.5 H Z$

\begin{tabular}{|c|c|c|c|}
\hline Amplitude (cm) & Change offset (degrees) & Circle Trajectory radius(m) & Speed of turning swimming (rad/s) \\
\hline \multirow{3}{*}{7.5} & 2 & 7.5 & $\pi$ \\
\cline { 2 - 3 } & 6 & 3.40 & \\
\cline { 2 - 3 } & 13 & 1.90 & \\
\cline { 2 - 3 } & 21 & 0.60 & \\
\hline 20.1 & 29 & 0.35 & $\frac{\pi}{3}$ \\
\hline
\end{tabular}

\subsection{Power Consumption Calculation}

The supply voltage of the control board was set at 5 Volt and the outputs of the servomotor are:

Torque 0.255 N.m, Speed6.98rad/s, Power1.78w. Battery Ni-Cd, Capacity $=700 \mathrm{mAh}$

Based on specifications of servomotor and the battery, the servo can operate up to 170minutes.

\subsection{Added Load at the Center of Gravity}

To test the capability of the robot in carrying additional equipment (camera, GPS, etc.) a different load was attached to the robot's center of gravity. With $\mathrm{f}=0.5 \mathrm{~Hz}$ and $\mathrm{A}=7.5 \mathrm{~cm}$, the forward velocity reduced as shown in table 4 .

Table3. Forward Swimming Speed with Additional Load

\begin{tabular}{|l|l|l|}
\hline Amplitude (cm) & \multicolumn{1}{|c|}{ Load (gram) } & Forward Swimming speed (cm/s) \\
\hline \multirow{4}{*}{7.5} & 100 & 12 \\
\cline { 2 - 3 } & 500 & 11 \\
\cline { 2 - 3 } & 900 & 9.8 \\
\cline { 2 - 3 } & 1300 & 9 \\
\cline { 2 - 3 } & 1700 & 7 \\
\cline { 2 - 3 } & 2100 & 5.8 \\
\cline { 2 - 3 } & 2500 & 4.5 \\
\cline { 2 - 3 } & 2980 & Sink \\
\hline
\end{tabular}

Figure7. Shows the rate of reduction of swimming speed by adding load to the center of mass of the robot. As it shown in graph 1, each kilogram causes, the reduction in speed by 15 percent.

\section{NUMERICAL EXAMPLE}

The weight of the robot is $1 \mathrm{~kg}$ and its length is $61 \mathrm{~cm}$. In this novel design, the tail was so flexible that it can be as similar as possible to the shape of a real fish's body. The tail actuation was produced by using a servomotor connected to the tail through cable strings. The robotic fish uses a $40 \mathrm{KHz}$ radio transmitter to control its forward swimming and steering. The turning movement is created by implying offset angle to the tail propulsion. This Robot has the capability to carry a maximum of $2.98 \mathrm{~kg}$ weight for extra accessories. The robotic fish was tested with different actuation frequencies and amplitudes. The maximum speed at $\mathrm{f}=1.25 \mathrm{HZ}$ and $\mathrm{A}=7.5 \mathrm{~cm}$ was $\mathrm{v}=0.188 \mathrm{~m} / \mathrm{s}$.

\section{Comparing SWimming SPEed}

We compared our model with three other robotic fishes with flexible tail from the literature. The results of this comparison is shown in table 5. (Other samples to compare are all submerge)

Table4. comparing swimming speed

\begin{tabular}{|c|c|c|c|}
\hline Robotic fish & Forward Swimming speed(BL/s) & Forward Swimming speed(cm/s) & Total length (cm) \\
\hline$[7]$ & 0.15 & 10 & 66 \\
\hline$[8]$ & 0.44 & 15 & 33.9 \\
\hline$[9]$ & 0.1 & 3 & 30 \\
\hline Float & 0.308 & 18.8 & 61 \\
\hline Submerge & 0.1 & 5.8 & 61 \\
\hline
\end{tabular}




\subsection{Effect of Changed Amplitude}

The robotic fish was tested for the displacement of the tip of the tail from $8 \mathrm{~cm}$ to $18 \mathrm{~cm}$ and fixed frequency of $1 \mathrm{~Hz}$. The results is shown in table 2 . As it can be seen in the table 2 , the speed increases by increasing the amplitude.

Table5. Forward swimming speed at various tail's displacement and $F=1 \mathrm{~Hz}$

\begin{tabular}{|c|c|}
\hline Amplitude(cm) & Forward Swimming Speed(cm/s) \\
\hline 4 & 11 \\
\hline 5.5 & 13.5 \\
\hline 7.5 & 18.1 \\
\hline 9 & 18.5 \\
\hline
\end{tabular}

Lighthillproposed that BCF flapping can be described by the traveling wave model below:

$$
y(x, t)=\left(c_{1} x+c_{2} x^{2}\right) \sin \left(\frac{2 \pi}{\lambda} x+\omega t\right)
$$

Where $y$ and $x$ are sideward and forward displacements respectively, $t$ is the time, $c_{1}$ and $c_{2}$ are the linear and quadratic wave amplitudes, $\lambda$ is the body wave length and $\omega$ is the wave frequency.

The type of swimming method of the robotic fish is based on BCF method, so the propulsive wave curve can be described by equation (2).

\section{2D HydRodyNAMIC SIMULATION MODEL}

\subsection{Dynamic Analysis of the Surrounding Fluid}

The 2D geometry and the tetrahedronal mesh of the robot fish is generated by GAMBIT. Figure 8 shows the top view of the meshing of the robot fish. In the first step of the design,the water flow and the dynamic analysis of the surrounding water is simulated by CFD (computational Fluid Dynamic). In the dynamic analysis of the water flow surrounding the fish robot, the speed of water flow was adjusted $14 \mathrm{~m} / \mathrm{s}$ and the robotic fish was fixed and the pressure, velocity and vorticity (Fig. 9,10,11) countors were analyzed. Given the smooth flow around the robotic fish and where the vortex is formed, it can be concluded that the geometrical shape of the robotic fish is efficient for swimming.

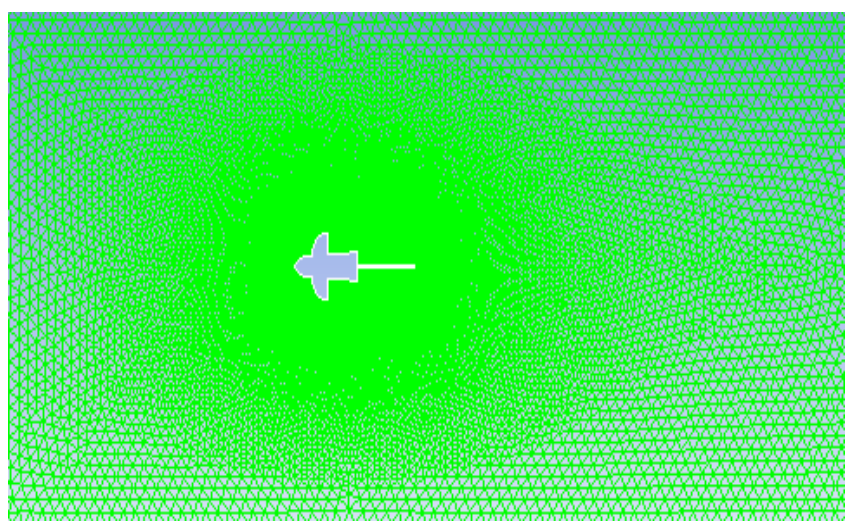

Figure8. The robot fish mesh

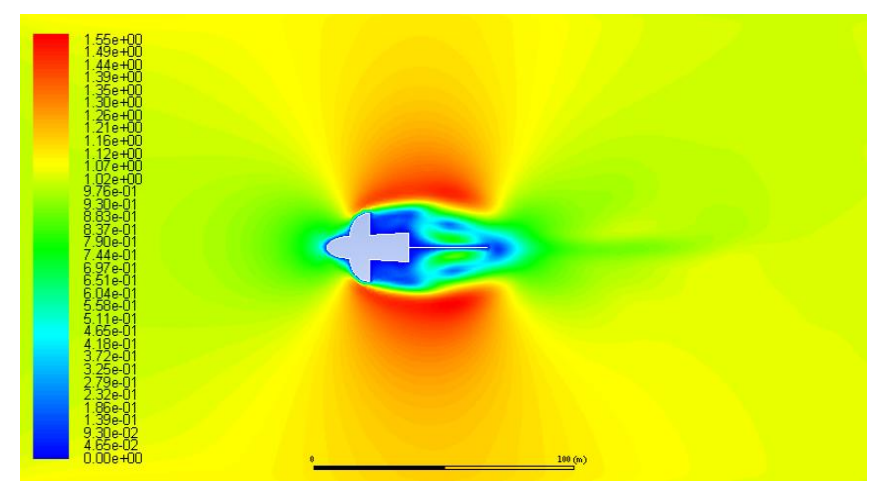

Figure9. The pressure countors 


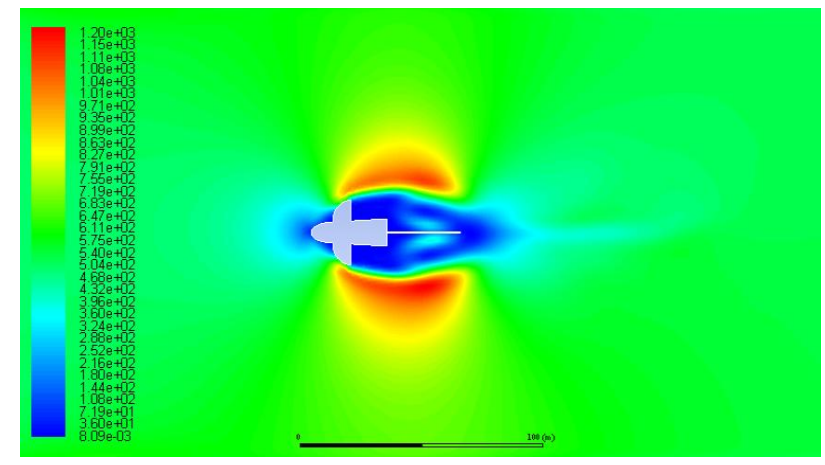

Figure10. The velocity countors

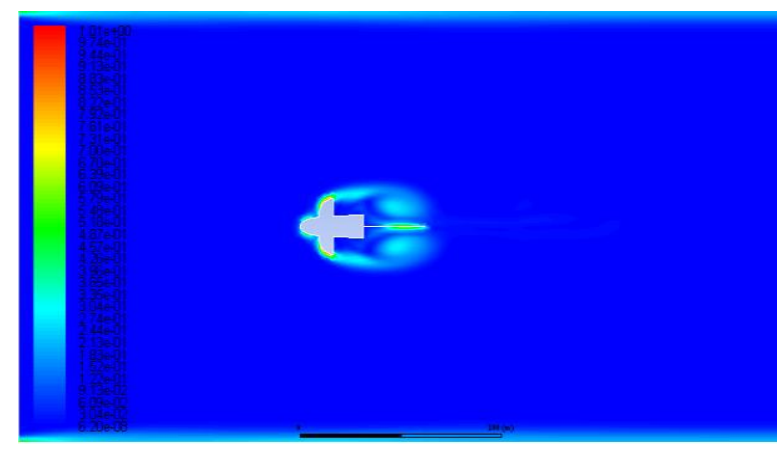

Figure11. The vortices countors

\subsection{Defination of the Swimming Movement of the Robot Fish}

At this stage, in ANSYS fluent the alternative movement of the fish robot was defined according to Eq.(2) where $c_{1}=0.03, c_{2}=0.07, \mathrm{k}=\frac{2 \pi}{\lambda}=0.35, \omega=2 \pi$ using UDF(USE-defined distribution) and also the swimming of the robotic fish was simulated using dynamic mesh. The swinging period of the oscillating movement of the fish tail in the simulations equals $1 \mathrm{~s}$. distribution of pressure surrounding the fish is shown in figure 12. These figures illustrate that low energy is needed to move the tail of the robotic fish.

\subsection{Pressure Distribution}

At $\mathrm{t}=0.25 \mathrm{~s}$ (Fig.12(a)) the tail of the fish start swinging from the extreme right side to the left the positive pressure is generated on the right side of the tail and it has a wilder distribution than the negative pressure on the left side. The focus of the positive pressure is mostly near the tip of the tail that generate trust along left-forward. The generated pressures are due to the pushed back water by the tail. Also, since the amplitude of the tail movement reduces from tip to the end. Therefore, the amount of positive pressure increases from the end of the tail to the tip and as a result the vortex is generated near the tip of the tail and a big amount of water is pushed towards the right side of the tail.

At $\mathrm{t}=0.75 \mathrm{~s}$ (Fig.12(b)) the whole process is reversed.

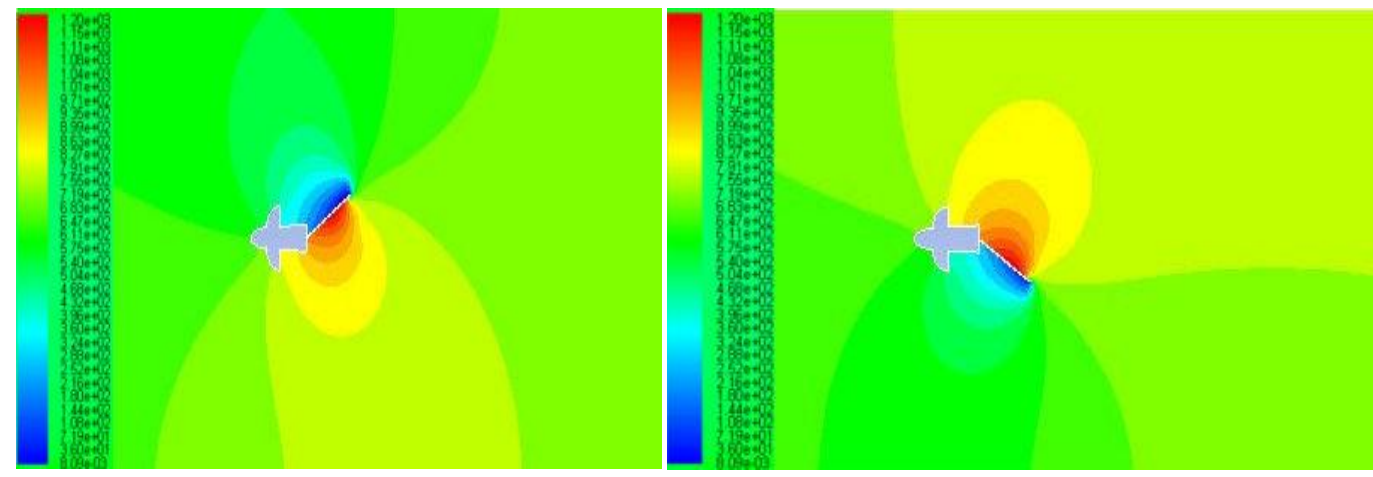

a. reaching the extreme right rotation position $(t=0.25 \mathrm{~s}) \mathrm{b}$. reaching the extreme left rotation position $(\mathrm{t}=0.75 \mathrm{~s})$

Figure12. The pressure distribution around the robot fish. 


\subsection{Velocity}

The velocity was analyzed at the same moment that the pressure was analyzed. The velocity countorare shown in figure 13.

At $\mathrm{t}=0.25 \mathrm{~s}$ (Fig.13(a)) the tail of the robotic fish is swinging from right to left and the vortex shads from the tip to the end counterclockwise that causes the generation of pressure from right to left. Therefore, the generated pressure contributes to the movement of the tail.

At $\mathrm{t}=0.75 \mathrm{~s}$ (Fig.13(b)) the whole process is reversed.

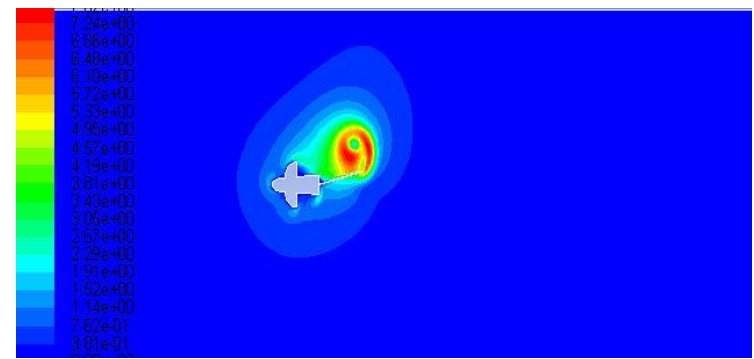

a. tail waving to the left $(\mathrm{t}=0.25 \mathrm{~s})$

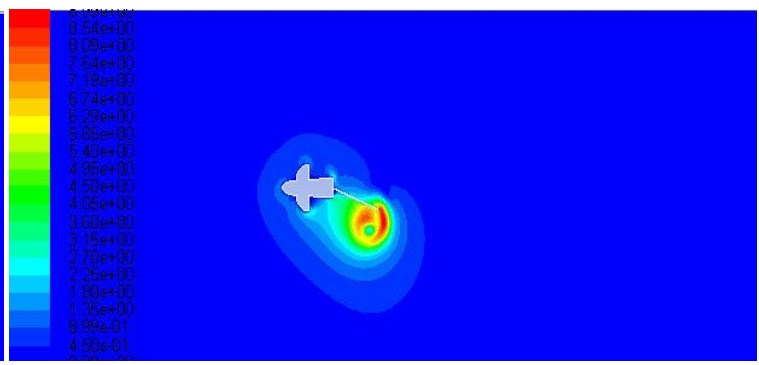

b. tail waving to the right $(\mathrm{t}=0.75 \mathrm{~s})$

Figure13. Velocity distribution

\subsection{Force}

The frequency of lateral motion of robotic tail in ANSYS is adjusted to 1 hertz and the drag and lift forces produced by the fish tail are analyzed (Fig.14).

Diagram A illustrates the peaks of lift coefficient in a full cycle of tail movement.

Diagram B shows drag coefficient in a full cycle of tail movement which is almost zero.

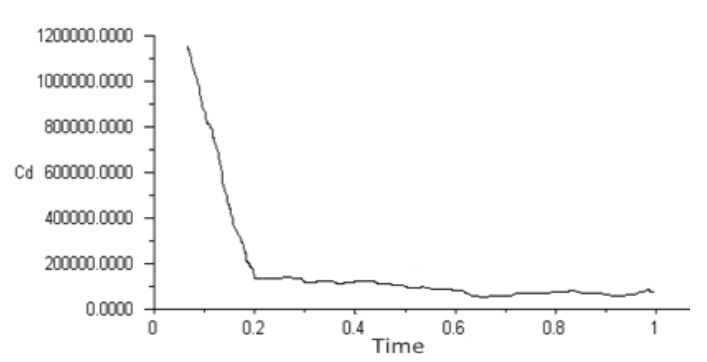

a. drag

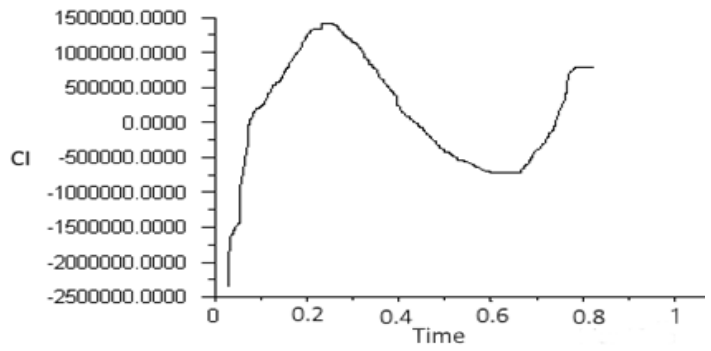

b. lift

Figure14. Calculated variations of drag and lift coefficients with movement of the tail.

\section{CONCLUSION}

A biomimetic robotic fish was designed and fabricated. The model was based on the body of the golden Trout that is categorized in carangiform swimmers. The swimming of the robotic fish was evaluated using CFD, the results of which played an important role to optimize the performance over the course of the design. based on the obtained results, the formation of the vortex and its effect on the lateral movement of the tail and the way it pushes the water that leads to the thrust, were explained. This robot was developed a simple and low cost design with an acceptable efficiency compared to the robots mentioned in the literature. Also the velocity and maneuverability of the fish while making a U-turn, was compared to the flexible tail ones and the result was successful.

The main application for this robotic fish is underwater surveillance and condition monitoring.

For this, the robot was equipped with a GoPro camera with remote control and wireless transmitting signal. The camera was activated and start recording video and capturing images when the robot reached the desired location and the output was observed trough the receiving device. The detail of this work and case studies would be discussed with details in the future paper.

For the future work, it is proposed to design an aerodynamic body to get better efficiency and speed also optimizing the tail thickness. In addition, adding the ability to this robot to dive and rise would be 
a fabulous idea. Also installing sensors like sonar, GPS and image processing unit (IPU) can be the next step to improve this model. Also optimizing the speed of tail's lateral movement can improve the swimming speed.

\section{ACKNOWLDGEMENT}

The authors wish to thank Dr. Zabihollah from Sharif University Int. campus for the practical support of the present work.

\section{REFERENCES}

[1] Masoomi, S. F., Haunholter, A., Merz, D., Gutschmidt, S., Chen, X., \& Sellier, M. (2014). Design, Fabrication, and Swimming Performance of a Free-Swimming Tuna-Mimetic Robot. Journal of Robotics, 2014, 1-7. http://doi.org/10.1155/2014/687985

[2] Zhou, C., Hou, Z., Cao, Z., Wang, S., \& Tan, M. (2013). Motion modeling and neural networks based yaw control of a biomimetic robotic fish. Information Sciences, 237, 39-48. http://doi.org/10.1016/j.ins.2011.02.015

[3] Du, R. (2015). Robot Fish. (R. Du, Z. Li, K. Youcef-Toumi, \& P. Valdivia y Alvarado, Eds.). Berlin, Heidelberg: Springer Berlin Heidelberg. http://doi.org/10.1007/978-3-662-46870-8

[4] Takada, Y., Koyama, K., \& Usami, T. (2014). Position Estimation of Small Robotic Fish Based on Camera Information and Gyro Sensors. Robotics, 3(2), 149-162.

[5] Thinh, N. T., Phuong, N. N., \& Tho, T. P. (2012). Autonomous inspection with multi-link robotic fish.

[6] Wu, Z., Yu, J., Tan, M., \& Zhang, J. (2014). Kinematic Comparison of Forward and Backward Swimming and Maneuvering in a Self-Propelled Sub-Carangiform Robotic Fish. Journal of Bionic Engineering, 11(2), 199-212. http://doi.org/10.1016/S1672-6529(14)60037-8.

[7] Katzschmann, R. K., Marchese, A. D., \& Rus, D. (2016). Hydraulic Autonomous Soft Robotic Fish for 3D Swimming. In M. A. Hsieh, O. Khatib, \& V. Kumar (Eds.), Intelligent Robots and Systems (IROS), 2011 IEEE/RSJ International Conference on (Vol. 109, pp. 405-420). Cham: Springer International Publishing. http://doi.org/10.1007/978-3-319-23778-7_27.

[8] Marchese, A. D., Onal, C. D., \& Rus, D. (2014). Autonomous Soft Robotic Fish Capable of Escape Maneuvers Using Fluidic Elastomer Actuators. Soft Robotics, 1(1), 75-87. http://doi.org/10.1089/soro.2013.0009.

[9] Rossi, C., Colorado, J., Coral, W., \& Barrientos, A. (2011). Bending continuous structures with SMAs: a novel robotic fish design. Bioinspiration \& Biomimetics, 6(4), 045005. http://doi.org/10.1088/1748$3182 / 6 / 4 / 045005$.

[10] Asgeirsson, S. (2013). Motion analysis for Arctic char and Atlantic salmon. University of Iceland.

[11] Nguyen, P. L., Do, V. P., \& Lee, B. R. (2013). Dynamic Modeling of a Non-Uniform Flexible Tail for a Robotic Fish. Journal of Bionic Engineering, 10(2), 201-209. http://doi.org/10.1016/S16726529(13)60216-4

[12] Leissa A W, Qaut M S. Vibration of Continuous Systems.

[13] McGraw-Hill Professional, NY, USA, 2011

Citation: Kasra Daryani Tabrizi, et.al, (2019)" Design and Fabrication and Hydrodynamic Analysis of a Fish Robot for Underwater Surveillance", International Journal of Modern Studies in Mechanical Engineering, 5(3), pp. 22-30. DOI: http://dx.doi. org/10.20431/2454-9711.0503004

Copyright: ( $) 2019$ Authors, This is an open-access article distributed under the terms of the Creative Commons Attribution License, which permits unrestricted use, distribution, and reproduction in any medium, provided the original author and source are credited. 